\title{
Acoustic and aerodynamic determinants of the sound quality of flutes
}

Neville H. Fletcher

Citation: The Journal of the Acoustical Society of America 95, 2858 (1994); doi: 10.1121/1.409536

View online: https://doi.org/10.1121/1.409536

View Table of Contents: https://asa.scitation.org/toc/jas/95/5

Published by the Acoustical Society of America

\section{ARTICLES YOU MAY BE INTERESTED IN}

Resonance and Sounding Frequencies of the Flute

The Journal of the Acoustical Society of America 40, 99 (1966); https://doi.org/10.1121/1.1910070

Effect of Material on Flute Tone Quality

The Journal of the Acoustical Society of America 49, 520 (1971); https://doi.org/10.1121/1.1912381

Manufacturing processes and their relationship to musical qualities of flutes

The Journal of the Acoustical Society of America 95, 2859 (1994); https://doi.org/10.1121/1.409488

Flute measurements in a Physics of Music lab

Proceedings of Meetings on Acoustics 20, 035004 (2013); https://doi.org/10.1121/1.4895818

Acoustical correlates of flute performance technique

The Journal of the Acoustical Society of America 57, 233 (1975); https://doi.org/10.1121/1.380430

Applying the reactive power approach to the woodwind instruments

The Journal of the Acoustical Society of America 95, 2859 (1994); https://doi.org/10.1121/1.409537

Advance your science and career as a member of the 
$3: 40$

2pEA6. Loss processes in piezoelectric PVDF and their effect on broadband transducers. D. A. Summa (Sound Solutions, 11718 Tallow Field Way, Austin, TX 78758-3520) and E. L. Hixson (Univ. of Texas at Austin, Austin, TX 78712)

Classic electromechanical models are extended to transducers fabricated from inherently lossy polymeric materials. To auginent sparse data, phenomenological models and molecular estimation techniques based upon structure-property relationships are used to examine relaxation processes and reconstruct elastic and dielectric loss tangent surfaces as a function of frequency, temperature, and pressure $(f, T, P)$. The improved material descriptions are used to assess the acoustic performance of a typical thickness-mode PVDF hydrophone over a range of environmental parameters of interest in sonar. Variations in elastic parameters shift the resonance frequency, cause a departure from a truly " $\rho c$-matched" condition, and introduce a dynamic variation in the open-circuit receive sensitivty. Variations in the dielectric parameters of similar magnitude occurring at the same $(f, T, P)$ coordinates ensure that the wideband response remains flat to within $1 \mathrm{~dB}$, depending upon the geometry of the device and operating parameters chosen. The effects of intrinsic material losses are most pronounced in the internal noise spectrum of the PVDF hydrophone. In warm waters noise levels average $10 \mathrm{~dB}$ lower than predicted by a constant loss model, whereas in cool waters noise levels are 6-10 dB below the predictions based upon these models at low frequencies $\left[O\left(10^{2}\right) \mathrm{Hz}\right.$ ], rising to $10-18 \mathrm{~dB}$ above the constant loss predictions at frequencies on the order of $10^{4}-10^{5} \mathrm{~Hz}$. [Partial support provided by the Independent Research \& Development Program, Applied Research Laboratories, The University of Texas at Austin, Austin, TX.]

2pEA7. Piezoelectric composite transducers. Leslie Bowen, Richard Gentilman, Hong Pham, and Daniel Fiore (Materials Systems, Inc., 521 Great Rd., Littleton, MA 01460)

Piezoelectric ceramic/polymer composites have found increasing use in high resolution medical ultrasound systems and advanced Navy underwater acoustic applications. Composite transducers offer design versatility and performance advantages over monolithic piezoelectric ceramic and monolithic piezoelectric polymer devices under both sensing and actuating conditions. In particular, composite transducers allow improved acoustic impedance matching to water and body fluids, improved lateral mode cancellation, enhanced thickness mode coupling coefficient, and naturally broad bandwidth. However, applications for these versatile transducers have been limited by the difficulties of costeffectively manufacturing the arrays of fine PZT ceramic elements required for composite assembly. At Materials Systems, Inc., ceramic injection molding has been developed into a viable net-shape process for making complex-shaped, fine scale PZT 1-3 and 2-2 transducer element arrays. This presentation describes the injection molding approach for manufacturing piezocomposite transducers, and reviews the sensing and actuating performance of injection molded 1-3 transducers over the frequency range $1 \mathrm{kHz}$ to $5 \mathrm{MHz}$. [This work is funded by ONR.]

\subsection{0}

2pEA8. Producing a distortion-free output from a PMN/PT transducer using large driving voltages. S. E. Forsythe and J. C. Piquette (Naval Res. Lab., Underwater Sound Reference Detachment, P. O. Box 568337, Orlando, FL 32856)

PMN/PT (lead magnesium niobate/lead titanate) is an electrostrictive ceramic that exhibits high strains when an unpoled sample is subjected to a high voltage. The strain as a function of voltage is an almost perfect square law, $s=k V^{2}$, over a large range of voltages, neglecting higher-order effects such as hysteresis and saturation. This response suggests that a transducer made of PMN/PT should be driven using an ac signal superimposed upon a large bias voltage. The bias voltage is needed to achieve a large effective strain/volt in response to the ac signal. If the ac signal amplitude is greater than a few percent of the bias voltage, significant distortion appears in the output due to the nonlinear response. To achieve the full potential response of this material, it is desirable to shape the driving signal such that the ac voltage swings over nearly twice the bias voltage while faithfully reproducing the desired signal at the output. Techniques for accomplishing this, even when the desired signal is complex or transient, by using models and their inverses are discussed and experimental results are presented. [Work supported by Office of Naval Research.]

\title{
Session 2pMU
}

\author{
Musical Acoustics: Air Reed Musical Instruments \\ Robert D. Collier, Chair \\ Department of Mechanical Engineering, Tufts University, Medford, Massachusetts 02155
}

Chair's Introduction-1:30

Invited Papers

1:35

2pMU1. Acoustic and aerodynamic determinants of the sound quality of flutes. Neville H. Fletcher (Res. School of Physical Sci. and Engineering, Australian Natl. Univ., Canberra ACT 0200, Australia)

After nearly 200 years of research, the precise nature of the aerodynamic driving mechanism of flutes and organ pipes is still incompletely determined. First-order theories using simplified acoustic approximations give reasonable agreement with experiment in terms of the dependence of acoustic output power and spectrum on the parameters available to the instrument maker and the player, but a detailed description needs to take account of aerodynamic phenomena at the level of individual vortices. A current version of the theory is presented and discussed in relation to the control of sound quality available to the flute player 
through selection of embouchure-hole size and shape and by variation of playing technique. New measurements on some of these quantities are presented.

2pMU2. Applying the reactive power approach to the woodwind instruments. Xavier Boutillon (Lab. d'Acoust. Musicale, Univ. Paris 6, Case 161, 4 place Jussieu, F75005 Paris, France)

The time-domain approach of self-sustained instruments such as the violin or the clarinet has sometimes revealed a marked hysteresis between force or pressure and velocity or flow. Formally defined by analogy with the active power, the reactive power $Q_{n}=n \pi p_{n} f_{n} \sin \left(\varphi_{n}\right)$ injected by a component into a pipe is highly sensitive to phase differences between pressure and flow components. The reed dynamics is one major cause for hysteresis; under various assumptions, the reed stiffness will be expressed as

$$
k=S^{2} \frac{\pi^{2}}{T} \frac{\Sigma_{n} p_{n}^{2} n^{2}}{\Sigma_{n} Q_{n}}=S^{2} \frac{\pi}{T} \frac{\Sigma_{n} p_{n}^{2} n^{2}}{\Sigma_{n}\left[n p_{n}^{2} \sin (\varphi n) /\left|Z_{n}\right|\right]} .
$$

In the absence of hysteresis, the cancellation of $Q$ is a generalization to a multimodal system of the usual condition $\sin (\varphi)=0$ that a simple oscillator fulfills when it vibrates at its resonance frequency. As the total reactive power $Q$ rapidly varies along with the playing frequency, the frequency range over which $Q$ can be canceled for various spectra might be related to the pitch-freedom range of the instrument. [Work supported by CNRS and the French Ministry of Culture.]

$$
\text { 2:40 }
$$

2pMU3. Experimental determination of the stiffness of a saxophone reed by means of the evaluation of the reactive power. Vincent Gibiat (Lab. Ondes et Acoust., ESPCI, 10 rue Vauquelin, F75005 Paris, France) and Xavier Boutillon (Univ. Paris 6, F75005 Paris, France)

A simple model for a saxophone or a clarinet reed—one pure spring with stiffness $k$-is acceptable for frequencies beneath the resonance frequency of the reed. When the instrument sustains a harmonic tone, Eq. (1) of the previous paper holds and the right-hand side quantities can be experimentally determined in order to evaluate $k$ or $k / S^{2}$. Experiments were driven on a saxophone so as not to encounter large uncertainties due to the weakness of even harmonics and corresponding minima of the input impedance of a cylindrical pipe. The reed stiffness is derived from separate measurements of the input impedance (TMTC method) and of the internal pressure spectrum when the saxophone is played by a musician or blown by an artificial system. Combined with an evaluation of the reed area, results on reed stiffness under playing conditions are in good agreement with compliance values measured on isolated reeds by other authors. The reactive power balance between the reed and the pipe also sheds a new light on the playing frequency changes that are observed when the saxophone is played softly or loudly without any mouth correction. A link with the anharmonicity of the pipe resonances will be shown.

2pMU4. Numerical fluid flow model for predicting playing frequencies. Peter L. Hoekje (Dept. of Phys., Univ. of Northern Iowa, Cedar Falls, IA 50614-0150)

A numerical model is designed in order to predict playing frequencies of air reed instruments. The air reed mechanism is modeled by dynamically solving the Navier-Stokes equation for an incompressible fluid over a two-dimensional grid in the vicinity of the jet outlet, the edge on which the jet impinges, and a short distance into the main bore. The rest of the main bore is modeled as a quasi-one-dimensional waveguide, represented by an impulse response function. At the junction between the two parts of the model, the exciting wavefront is typically bulged, so the velocity distribution must be integrated over the bore cross section in order to calculate the excitation of the main bore. The results confirm that mode locking and octave overblowing occur in an instrument whose frequency response has nearly harmonic resonances, but not in one whose second resonance is much higher than twice the first resonance frequency. Comparisons of the resulting playing frequencies with experimental playing frequencies, and with those calculated by a simple passive model, will be presented.

\section{3:30}

2pMU5. Manufacturing processes and their relationship to musical qualities of flutes. Steven A. Wasser (Vern Q. Powell Flutes, Inc., 257 Crescent St., Waltham, MA 02154)

Manufacturers of musical instruments are under constant pressure to both improve productivity and enhance the performance qualities of their products. However, the higher the perceived musical quality of an instrument, the more difficult it becomes to implement technological changes. For example, apparent improvements in materials and/or the manufacturing process may have subtle effects which could adversely affect particular performance qualities which characterize the manufacturer's instrument. It is known that some imperfections in materials and manufacturing processes may actually enhance the artist's musical abilities. Therefore, it becomes the responsibility of the manufacturer to establish a dialogue between the subjective artistic requirements of the musician and the relatively objective manufacturing factors such as material properties, dimensions, and processes. The issues of how a manufacturer of a distinctive, traditional handcrafted flute (with a loyal following among professional flutists) approaches the design and manufacture of a new flute to meet the needs of a wider range of players will be discussed. Particular emphasis is placed on the steps taken to maintain the family relationship between the musical qualities of the new flute and those of the traditional one and, at the same time, to ensure sufficient differences in performance capabilities in order to maintain a clear distinction between the two instruments. 